\title{
Sensitivity of computed tomography performed within six hours of onset of headache for diagnosis of subarachnoid haemorrhage: prospective cohort study
}

\author{
Jeffrey $\mathrm{J}$ Perry associate professor of emergency medicine and of epidemiology and community \\ medicine ${ }^{1}$, Ian G Stiell professor and chair department of emergency medicine ${ }^{1}$, Marco L A Sivilotti \\ associate professor of emergency medicine and of pharmacology and toxicology ${ }^{2}$, Michael J Bullard \\ professor of emergency medicine ${ }^{3}$, Marcel Émond assistant professor ${ }^{4}$, Cheryl Symington research \\ coordinator $^{1}$, Jane Sutherland research coordinator ${ }^{5}$, Andrew Worster associate professor ${ }^{6}$, Corinne \\ Hohl assistant professor ${ }^{7}$, Jacques S Lee assistant professor ${ }^{8}$, Mary A Eisenhauer associate \\ professor $^{9}$, Melodie Mortensen research coordinator ${ }^{1}$, Duncan Mackey associate professor ${ }^{3}$, Merril \\ Pauls associate professor ${ }^{10}$, Howard Lesiuk consulting neurosurgeon ${ }^{11}$, George A Wells professor \\ biostatistics and epidemiology ${ }^{12}$

\footnotetext{
${ }^{1}$ Department of Emergency Medicine, University of Ottawa, Clinical Epidemiology Program, Ottawa Hospital Research Institute, Ottawa, ON, Canada; ${ }^{2}$ Departments of Emergency Medicine and of Pharmacology and Toxicology, Queen's University, Kingston, ON ; ${ }^{3}$ Department of Emergency Medicine, University of Alberta, Edmonton, AB ; ${ }^{4}$ Department of Emergency Medicine, Université Laval, QC ; ${ }^{5}$ University of Ottawa, Clinical Epidemiology Program, Ottawa Hospital Research Institute, Ottawa, ON; ${ }^{6}$ Department of Emergency Medicine, University of British Columbia, Vancouver, BC ; ${ }^{7}$ Division of Emergency Medicine, McMaster University, Hamilton, ON ; ${ }^{8}$ Division of Emergency Medicine, University of Toronto, Toronto, ON ; ${ }^{9}$ Division of Emergency Medicine, University of Western Ontario, London, ON $;{ }^{10}$ Department of Emergency Medicine, University of Manitoba, Winnipeg, MB; ${ }^{11}$ Division of Neurosurgery, University of Ottawa, the Clinical Epidemiology Program, Ottawa Hospital Research Institute, Ottawa, $\mathrm{ON} ;{ }^{12}$ Department of Epidemiology and Community Medicine, University of Ottawa, Clinical Epidemiology Program, Ottawa Hospital Research Institute, Ottawa, ON
}

\begin{abstract}
Objective To measure the sensitivity of modern third generation computed tomography in emergency patients being evaluated for possible subarachnoid haemorrhage, especially when carried out within six hours of headache onset.

Design Prospective cohort study.

Setting 11 tertiary care emergency departments across Canada, 2000-9. Participants Neurologically intact adults with a new acute headache peaking in intensity within one hour of onset in whom a computed tomography was ordered by the treating physician to rule out subarachnoid haemorrhage.

Main outcome measures Subarachnoid haemorrhage was defined by any of subarachnoid blood on computed tomography, xanthochromia in cerebrospinal fluid, or any red blood cells in final tube of cerebrospinal fluid collected with positive results on cerebral angiography.

Results Of the 3132 patients enrolled (mean age 45.1, 2571 (82.1\%) with worst headache ever), 240 had subarachnoid haemorrhage (7.7\%). The sensitivity of computed tomography overall for subarachnoid
\end{abstract}

haemorrhage was $92.9 \%$ (95\% confidence interval $89.0 \%$ to $95.5 \%$ ), the specificity was $100 \%(99.9 \%$ to $100 \%)$, the negative predictive value was $99.4 \%$ (99.1\% to $99.6 \%)$, and the positive predictive value was $100 \%$ (98.3\% to 100\%). For the 953 patients scanned within six hours of headache onset, all 121 patients with subarachnoid haemorrhage were identified by computed tomography, yielding a sensitivity of $100 \%$ (97.0\% to $100.0 \%$ ), specificity of $100 \%$ (99.5\% to $100 \%)$, negative predictive value of $100 \%$ (99.5\% to $100 \%$ ), and positive predictive value of $100 \%(96.9 \%$ to $100 \%)$.

Conclusion Modern third generation computed tomography is extremely sensitive in identifying subarachnoid haemorrhage when it is carried out within six hours of headache onset and interpreted by a qualified radiologist.

\section{Introduction}

Headache is a common symptom and accounts for about $2 \%$ of presenting complaints in the emergency department. ${ }^{1} \mathrm{~A}$ foremost consideration in these patients is the exclusion of spontaneous subarachnoid haemorrhage, a neurosurgical emergency identified 
in about $1 \%$ of patients with headache in the emergency department. ${ }^{2}$ The overall mortality of subarachnoid haemorrhage is high, with $25-50 \%$ of patients dying within six months. ${ }^{3}$ About a third of survivors are left with neurological deficits affecting their activities of daily living. ${ }^{3}$ The prompt diagnosis of subarachnoid haemorrhage is particularly important in patients who present with normal neurological findings. Such patients can be difficult to diagnose and yet have the most to lose by a missed diagnosis. ${ }^{4}$ Moreover, nearly half of all patients with a subarachnoid haemorrhage have normal results on neurological examination and normal vital signs at initial presentation.

To exclude subarachnoid haemorrhage, traditional teaching says that patients should undergo a lumbar puncture if the initial computed tomogram yields negative results. ${ }^{5-9}$ Lumbar puncture, however, causes pain and delays discharge from the emergency department and can lead to postdural puncture headache. ${ }^{10}$ Most studies that evaluated the sensitivity of computed tomography for subarachnoid haemorrhage were retrospective reviews and found that sensitivity deteriorates rapidly over time because of erythrocyte dissipation and lysis. ${ }^{11-16}$ These prospective studies were small or used older computed tomography technology, used 12 or 24 hours as a cut off point, and found sensitivities ranging from $90.5 \%$ to $100 \% .^{13-15}{ }^{17-20}$ There have been no large prospective studies of emergency department patients with acute headache involving modern, multi-detector row third generation scanners (that is, $\mathrm{x}$ ray emitter and detector rigidly linked and operating in rotate/rotate geometry; multiple rows of detectors in $\mathrm{z}$ axis). ${ }^{21}$

We determined the sensitivity of modern computed tomography for identifying subarachnoid haemorrhage in neurologically intact patients who present to the emergency department with acute headache, especially when scans are performed within six hours of headache onset.

\section{Methods}

\section{Design and setting}

This prospective multicentre cohort study was conducted in the emergency departments of 11 university affiliated tertiary care teaching hospitals in Canada, November 2000 to December 2009. Study sites had a mean of 52000 emergency visits a year (range $39000-65000$ ) and a mean of 445 inpatient beds (range 289-622). Nine of the 11 sites had neurosurgical units. The duration of participation in the study varied across sites, with a mean of 4.2 years (range 1-9 years). During the study, sites were added based on additional funding, two sites dropped out because of relocation of key study staff, and in two sites the study was terminated because of low recruitment (that is, they enrolled less than $70 \%$ of eligible patients). This study was part of a larger project on clinical decision rules for patients with acute headache. ${ }^{22}$

\section{Study population}

We enrolled consecutive alert patients aged over 15 who presented with non-traumatic acute headache or with syncope associated with headache and underwent emergency head computed tomography as part of their diagnostic investigation. We defined "alert" as a Glasgow coma score of 15 (scale ranges from 3 to15, with 15 being normal), "non-traumatic" as no falls or direct trauma to the head in the previous seven days, and "acute" as headache reaching maximum intensity in less than one hour after onset. ${ }^{23}$ We excluded patients with onset of headache more than 14 days previously; recurrent headaches (three or more headaches of similar character and intensity as presenting headache); transfer from another hospital with confirmed subarachnoid haemorrhage; focal neurological deficits; papilloedema; or history of subarachnoid haemorrhage, aneurysm, ventricular shunt, or brain neoplasm. Computed tomography was ordered at the discretion of the treating physician, who was aware of the clinical decision rule study but was advised not to alter usual care because of the study.

\section{Data collection}

During the study, registration clerks, nurses, or treating physicians were asked to identify potentially eligible patients. The treating physician completed the clinical decision rule study data forms. At all study sites, a local study nurse manually reviewed every visit for headache to identify potentially eligible patients who might have been missed. If it was not clear from the clinical care notes that the patient was ineligible, they were deemed "missed eligible" even though this would probably overstate the number of patients missed because of poor charting.

Attending physicians certified in emergency medicine or supervised residents in an emergency medicine training programme assessed all patients. Treating physicians recorded the time of headache onset on to study data forms. The time of computed tomography was retrieved by local study staff based on radiology time stamps. We selected a priori an interval of six hours from headache onset to computed tomography as determined by our consensus group, consisting of five emergency physicians and one neurosurgeon, based on the available evidence and on temporal trends towards prioritising neuroimaging and technical improvements in imaging.

\section{Outcome measures}

Patients were deemed to be positive for subarachnoid haemorrhage if they had any of subarachnoid blood identified on unenhanced head computed tomography; visible xanthochromia in the cerebrospinal fluid; or red blood cells $\left(>5 \times 10^{6} / \mathrm{L}\right)$ in the final tube of cerebrospinal fluid collected and an aneurysm identified on cerebral angiography (digital subtraction, computed tomography, or magnetic resonance angiography). ${ }^{24}$

Qualified local radiologists (a neuroradiologist or general radiologist who routinely reports head computed tomography images), who were blinded to the study and data forms but who had routine clinical information, interpreted all computed tomograms. These radiologists were completely unaware of the study and reported the scans as usual. The final local "sign off" report was used, even though it might be created only the next day, especially when the scan was obtained during the evenings or weekends. Patients were typically managed according to the initial interpretation by the emergency physician or a radiology trainee at some sites, also in accordance with usual local care. Patients with a discordant radiology report were recalled. All computed tomography scanners were third generation, multi-slice scanners (from 4 to 320 slices/rotation). The protocols at the beginning of the study (2000-2) used $5 \mathrm{~mm}$ slices for the posterior fossa and $10 \mathrm{~mm}$ for the remainder of the brain. Since 2002, all sites adopted 5-7.5 mm cuts for the brain with $2.5-5 \mathrm{~mm}$ for the posterior fossa. Lumbar puncture was performed at the discretion of the treating physician, with consent from the patient, according to usual practice. Local laboratory technicians unaware of the study assessed the cerebrospinal fluid for xanthochromia by visual comparison against white paper. ${ }^{12} 1425$

Because the study did not interfere with usual clinical practice, many participants did not undergo lumbar puncture after normal 
results on computed tomography. Unless patients had a definitive cause for the headache identified on neuroimaging (such as subarachnoid haemorrhage, subdural haemorrhage, intracerebral haemorrhage, tumour with mass effect, meningitis, acute or subacute infarction) or had negative results on lumbar puncture (that is, no erythrocytes and no xanthochromia), they were followed for six months to ascertain their outcome. These patients underwent a structured telephone interview, and we reviewed hospital records at one and six months to identify any missed subarachnoid haemorrhages. Patients who had angiography without lumbar puncture underwent the full follow-up procedure. Patients requiring follow-up were called up to five times at varying times on different days of the week. For patients whom we could not reach by telephone, we attempted to ascertain their vital status by searching records from regional neurosurgical centres at the end of the study. Finally, we searched the provincial coroner's records for the names of all patients not known to be alive and free from subarachnoid haemorrhage at six months based on this passive follow-up of hospital records. By law, all sudden and unexpected deaths in Ontario are reported to the coroner, who has the authority to investigate and identify the cause of death.

\section{Analysis}

Data were sent by courier to the coordinating centre at the Ottawa Hospital Research Institute, entered into a SAS (SAS Institute, Cary, NC) database by trained data entry personnel, and verified by searching for outliers. We calculated sensitivity, specificity, negative likelihood ratios, and negative predictive values of computed tomography for subarachnoid haemorrhage, with $95 \%$ confidence intervals. At the end of the study we performed a sensitivity analysis to assess the differences between the patients who did undergo lumbar puncture and those who did not.

\section{Sample size}

We anticipated that, for a life threatening problem such as subarachnoid haemorrhage, clinicians would expect $100 \%$ sensitivity with a narrow confidence interval (such as $97 \%$ to $100 \%) .{ }^{26}$ Thus, we estimated that we required over 100 patients with subarachnoid haemorrhage in whom computed tomography was performed in less than six hours from onset of headache. With an overall prevalence of subarachnoid haemorrhage of about $7 \%$ in our previous work of similar patients and a median delay to imaging of six hours for positive subarachnoid cases (that is, in half of positive cases imaging was carried out within six hours of onset of headache), we estimated we required a total sample size of about 2860 patients.

\section{Results}

We enrolled 3132 patients, including 240 (7.7\%) with confirmed subarachnoid haemorrhage, from among 5424 potentially eligible patients with headache who presented to the study sites during our study (figure).

Tables 1 and 2 list the characteristics of our study cohort. Overall, patients were young (mean age $<45$ ), most $(82 \%)$ characterised the headache as being their worst ever, a quarter arrived by ambulance, half underwent lumbar puncture after negative results on computed tomography, and $240(7.7 \%)$ had subarachnoid haemorrhage. In $27(0.9 \%)$ patients the diagnosis was serious: $17(0.5 \%)$ had other haemorrhagic strokes, nine $(0.3 \%)$ had a brain tumour, and one $(0.03 \%)$ had bacterial meningitis.
For all study patients, the sensitivity of computed tomography overall was $92.9 \%$ (95\% confidence interval $89.0 \%$ to $95.5 \%$ ), the specificity was $100 \%$ (99.9\% to $100 \%)$, the negative predictive value was $99.4 \%$ (99.1\% to $99.6 \%$ ), and the positive predictive value was $100 \%$ (98.3\% to 100\%) (table 3). In four additional patients, classified as not having subarachnoid haemorrhage, the initial computed tomogram indicated an aneurysm without haemorrhage, and they underwent angiography. One patient with a giant aneurysm (>25 mm) underwent surgery for the aneurysm, and the three others (aneurysm size 2-5 mm) were managed conservatively. The sensitivity was $100 \%$ (97.0\% to $100.0 \%$ ) for subarachnoid haemorrhage in the 953 patients in whom computed tomography was performed within six hours of headache onset, with a specificity of $100 \%(99.5 \%$ to $100 \%)$, a negative predictive value of $100 \%(99.5 \%$ to $100 \%)$, and a positive predictive value of $100 \%$ (96.9\% to $100 \%$ ) (table 3 ). When computed tomography was performed more than six hours after headache onset, 17 of 119 patients with subarachnoid haemorrhage were not identified by unenhanced computed tomography (sensitivity $85.7 \%, 78.3$ to $90.9 \%$ ). For these 17 patients with a false negative result, computed tomography was performed from eight hours to eight days after the onset of headache. Of these 17 patients, 13 were diagnosed by xanthochromia in the cerebrospinal fluid on visual analysis and two by red blood cells in the final tube of cerebrospinal fluid with abnormal cerebral angiography. One patient had negative results on computed tomography 96 hours after the onset of headache without lumbar puncture; the patient returned to the emergency department and repeat computed tomography showed a new subarachnoid haemorrhage secondary to vertebral artery dissection. Another patient had an aneurysm identified, without subarachnoid haemorrhage, on the unenhanced computed tomogram; this was confirmed with angiography and it subsequently bled while the patient was awaiting surgery. Only six of these 17 underwent neurosurgical intervention (ventricular drain, aneurysm coiling or clipping). Of the 11 remaining cases, no cause for bleeding was found in 10, and one other was thought to have bleed secondary to a brain tumour. One patient with no source of bleeding identified had a $5 \mathrm{~mm}$ middle cerebral artery aneurysm, thought to be incidental, and the patient was alive and well at six months with conservative management.

There were 1931 patients requiring follow-up. Of these we contacted 1506 patients by telephone six months or more after their initial emergency visit; none was identified as having had subarachnoid haemorrhage. Of the remainder, we identified eight patients who died within six months after their index visit, all from other causes: five from cancer, one from pneumonia, one from an ischaemic stroke, and one presumed cardiac (patient had had a previous myocardial infarction and died 32 days after their visit for headache, but four days after a visit for chest pain; no autopsy was performed). Fifty patients (13 in the group who underwent computed tomography within six hours) could not be reached by telephone and had no subsequent hospital visits. None of these patients was seen at the regional neurosurgical referral centre within six months of the index visit, and they have been classified as negative for subarachnoid haemorrhage. For 31 of these patients, at the five Ontario sites, we were able to search provincial coroner reports. None of the 31 lost to all follow-up had reportedly died suddenly and unexpectedly in the province.

In our study, emergency physicians initially misinterpreted the computed tomography as normal in three cases and discharged these patients home. These three patients were recalled after review of the imaging by radiologists. All three underwent 
computed tomography more than six hours after the onset of headache. Another computed tomogram was also initially misinterpreted as normal by both the emergency physician and a radiology trainee. This patient presented four and a half hours after the onset of headache, had blood in the cerebrospinal fluid (632 $000 \times 10^{6} / \mathrm{L}$ in the fourth tube) attributed to a traumatic lumbar puncture by a neurosurgical trainee, and was found to have an aneurysm on follow-up magnetic resonance imaging angiogram five days later. In retrospect, the local neuroradiologist re-interpreted the initial scan as positive for subarachnoid haemorrhage. The patient underwent coiling and had a good clinical outcome.

Because many patients did not undergo lumbar puncture, we conducted a post hoc subgroup analysis of patients who underwent lumbar puncture versus those who did not. There was no significant difference in sex, loss of consciousness, arrival by ambulance, onset with exertion, vomiting, mean blood pressure, or heart rate. There were differences in mean age (higher chance of lumbar puncture in younger patients (mean age $47.1 v 43.0$ ), time to peak pain intensity (if faster peak (mean $5.7 v 9.4$ minutes)), neck pain (if present $(39.0 \% \vee 34.9 \%)$ ), and being characterised as the "worst headache ever" (if present $(91.6 \% v 76.3 \%)$ ) between the lumbar puncture and the no lumbar puncture groups.

There were 2292 eligible patients who were potentially eligible but were not enrolled because no data form was completed. This group of patients had similar characteristics to enrolled patients (mean age 45.3; 60\% women), and there were 137 subarachnoid haemorrhages (6\%). Of these, 67 patients with subarachnoid haemorrhage underwent computed tomography within six hours; all 67 had subarachnoid blood identified on unenhanced computed tomography.

\section{Discussion}

In this large prospective multicentre study we found that modern computed tomography technology is highly sensitive for subarachnoid haemorrhage when the scan is carried out within six hours of headache onset and when it is interpreted by a qualified radiologist. More than half of all cases of low grade subarachnoid haemorrhage were imaged within this time frame, and all were identified by plain computed tomography imaging. When computed tomography imaging is obtained more than six hours after headache onset, clinicians should continue to be cautious because of the decreasing sensitivity for subarachnoid haemorrhage beyond this time point.

\section{Comparison with previous studies}

Most previous studies have reported imperfect sensitivity for computed tomography when it is carried out over a longer time window. Boesiger and Shiber reported $100 \%$ sensitivity in a retrospective study with 177 patients with undifferentiated headache, but the cohort included only six cases of subarachnoid haemorrhage..$^{18}$ Other recent studies have had retrospectively estimated sensitivities of 93-98\%. ${ }^{14} 17{ }^{19}{ }^{20}$ A systematic review assessed the sensitivity of computed tomography for subarachnoid haemorrhage based on two studies and found that the sensitivity of head computed tomography for subarachnoid haemorrhage was $93 \%$ (95\% confidence interval $66 \%$ to $98 \%$ ). ${ }^{27}$ An older prospective study, using older second generation computed tomography technology, found an overall sensitivity of head computed tomography for subarachnoid haemorrhage of $90.5 \%$ within 24 hours of headache onset; however, when it was restricted to patients who were neurologically intact, the sensitivity was only $80 \% .{ }^{15}$ Finally, Cortnum and colleagues carried out a retrospective study of patients with confirmed subarachnoid haemorrhage. ${ }^{28}$ They identified only one patient who did not have positive results on computed tomography. This patient had delayed imaging (five days after the onset of headache) and required lumbar puncture. Our study differs in that we restricted our population to neurologically intact patients and shortened the cut off point to six hours after onset of headache. We found a much higher sensitivity, probably because of a shorter time delay to imaging and newer multi-slice scanners, which incorporate better resolution and thinner slice protocols.

\section{Clinical and policy implications}

The few cases of initially misinterpreted computed tomograms illustrate the importance of having a qualified radiologist with a high level of skill interpreting the head scans in a timely manner. We believe, in this era of digital imaging and remote reading, that early interpretation of head computed tomography by neuroradiologists should be widely available. Moreover, given the deterioration of the sensitivity of computed tomography over hours, we believe that urgent evaluation and imaging of emergency patients presenting with acute headache should be prioritised in keeping with a "brain attack" paradigm. Likewise, it is important that patients undergo imaging quickly after arrival to allow for a larger proportion of patients to be imaged within the six hour window.

It is important to note that the improved sensitivity of computed tomography with early imaging might reflect two important considerations. Firstly, as is well acknowledged, a relatively small volume of blood released into the subarachnoid space will diffuse away from the source of bleeding and haemolyse within hours, rendering computed tomography less able to distinguish the blood from cerebrospinal fluid as time passes. Secondly, time to imaging depends on multiple factors that will be influenced by the perceived severity of the headache. In other words, a patient with a history more concerning for subarachnoid haemorrhage (for example, older, presentation by ambulance, seems unwell, abnormal vital signs ${ }^{22}$ ) is more likely to be seen quickly and have neuroimaging prioritised, especially in an overcrowded emergency department. Thus, our observed improved sensitivity of computed tomography might reflect, to some degree, confounding by severity as these patients might have a larger volume subarachnoid haemorrhage than patients in whom initial assessment and imaging are delayed. The only slightly higher prevalence of subarachnoid haemorrhage (12\%) in the group seen in less than six hours suggests any such bias was in fact minor and does not undermine the recommendation to expedite time to computed tomography for all patients with headache in whom subarachnoid haemorrhage is a diagnostic consideration.

With an extremely high sensitivity when performed within six hours, an early unenhanced computed tomography can be considered a "rule out" test for subarachnoid haemorrhage. This observation challenges the dogma of performing a lumbar puncture in all patients despite a negative result on computed tomography. The strength of this conclusion necessarily hinges on the final outcomes of a small number of patients lost to follow-up. While we did achieve a high degree of certainty in our outcome classification, nearly $2 \%$ of patients were lost to all active follow-up at six months. Our study sites included the regional neurosurgical referral centres and would have identified any subsequent non-fatal subarachnoid haemorrhage. Moreover, nearly three quarters of patients who could not be contacted at six months were known to be alive and free from subarachnoid haemorrhage at least one month after the initial visit, a time 
within which most patients with a missed sentinel bleed or small subarachnoid haemorrhage would experience a re-bleed.

\section{Limitations}

Our planned follow-up procedure failed to identify a single case of missed subarachnoid haemorrhage resulting in death or diagnosed on a subsequent unscheduled visit. We recognise that such cases do occur and might be more common in smaller community hospitals (not represented in this study) where access to computed tomography imaging and to specialists is difficult. ${ }^{29}$ Even if we adopt a highly conservative view that a quarter of our patients lost to all follow-up could have experienced a subarachnoid haemorrhage, diagnosed and treated in a different region, or a sudden out of hospital death that was not reported to the coroner, the corresponding negative likelihood ratio for a computed tomography performed within six hours rises to only 0.024 (0.007 to 0.07$)$. Such a likelihood ratio could be incorporated into the informed discussion surrounding the risks and benefits of lumbar puncture after negative results on computed tomography for this diagnosis. After enrolling patients at multiple large hospitals across Canada for nearly a decade, and having failed to identify a single false negative, we are confident that such an event is indeed rare.

Our study is also limited by the absence of a single widely accepted standard criterion for subarachnoid haemorrhage. While we recognise that there is some controversy in the optimal method of detecting subarachnoid haemorrhage, there is no strong evidence to demonstrate the superiority of xanthochromia by spectrophotometer versus visual detection of xanthochromia plus red blood cell analysis. The specificity of

spectrophotometry defined xanthochromia is poor, and it is thus not discriminating for subarachnoid haemorrhage. As we mentioned, there is a lack of a standard to define subarachnoid haemorrhage, and we feel that our a priori consensus definition of positive blood on computed tomography, visual xanthochromia, or red blood cells in the final tube of cerebrospinal fluid with abnormal cerebral angiography is the most robust definition at present. We went further than this in that we conducted telephone and medical record follow-up at one and six months to further ensure that we did not miss any patients with subarachnoid haemorrhage. Finally, spectrophotometry was not available at our study centres (it is not available in $97 \%$ of hospitals in North America). ${ }^{30} \mathrm{We}$ believe that our composite definition is the best definition available at this time.

Some potentially eligible patients were not enrolled in this study because forms had not been completed. It is likely that because of our methods this number is somewhat artificially inflated. For example, when the time interval from onset to peak headache intensity was not explicitly charted, we were obliged to consider the patient as potentially eligible and thereby included as missed those patients recorded as having gradual onset headaches.

We asked physicians not to alter their practice and excluded many patients in whom no computed tomography was performed. Forcing physicians to order a test would have exposed patients to ionising radiation and increased healthcare costs. Similarly, not all patients with normal results on computed tomography underwent lumbar puncture. Overall, the group of patients undergoing lumbar puncture was a slightly higher risk group than those without. The impact of these differences on our final results is probably modest given that almost all of the patients who did not undergo computed tomography or lumbar puncture, or both, were alive at one and six month follow-up. ${ }^{22}$

\section{Conclusions}

This prospective study of patients with acute headache found that computed tomography was highly sensitive for subarachnoid haemorrhage, if it was performed with a modern multi-row detector third generation scanner with thin slices within six hours of headache onset and if the scans were interpreted by a qualified radiologist. We believe that these findings are robust enough to inform the clinical decision as to whether to perform lumbar puncture after negative results on computed tomography obtained under such conditions.

We thank the hundreds of physicians who completed our data collection forms and the emergency department nurses and clerks at the 11 study sites for their cooperation with the study; Albert E Lauwers, deputy coroner, and the Office of the Coroner of Ontario for their assistance verifying outcomes; and the following research staff at the study hospitals: Jan Buchanan (Vancouver General Hospital, Vancouver, BC), Evelyn Gilkinson (London Health Sciences Centre, London, ON), Erica Battram, Juanita Wilzer, Nicole Fortin, Renée Labreche (Ottawa Hospital-Civic Campus and General Campuses, Ottawa, ON), Harris Lari, Leslie Saunders, Ginny Willis, Sandy Sandilands (University of Alberta, Edmonton, Alberta), Deborah Wright, Johanna Pak (Sunnybrook and Women's College Health Sciences Centre, Toronto, ON), Kathy Bowes, Julie Richard, Deborah Crosby, Jane Reid, Nicholas Martin (Kingston General Hospital, Kingston, Ontario), Patricia Chabot (Hôpital de L'Enfant-Jésus, Quebec City, QC), Christina Brean (Hamilton Health Sciences Centre, Hamilton, ON), Marlene Myles (Lethbridge Regional Hospital, Lethbridge, AB), Irene Osinchuk (Winnipeg Health Sciences Centre, Winnipeg, MB). We also thank our colleagues at the Ottawa Hospital Research Institute (Sarai Cohn-Kalter, Malaika Mvungi, Sheryl Domingo, My-Linh Tran, Irene Harris, and Angela Marcantonio) for their assistance with this project.

The study was presented at the Society for Academic Emergency Medicine, New Orleans, Louisiana, May 2009, and the Canadian Association of Emergency Physicians, Calgary, Alberta, June 2009. Contributors: JJP developed and contributed substantially to study concept and design and secured research funding. All authors participated in the study design, supervised recruitment of patients and management of data, supervised the conduct of the trial and data collection, drafted the manuscript and/or contributed to its revision, and approved the final version. JJP, IGS, and GAW provided statistical advice and data analysis. JJP is guarantor.

Funding: The study was funded by the Canadian Institutes for Health Research (grants 67107, 153742), the Ontario Ministry of Health and Long Term Care, and the physicians of Ontario through the Physician's Services Incorporated Foundation (01-39). JJP is supported by a Canadian Institutes for Health Research new investigator award and was previously supported as a career scientist by the Ontario Ministry of Health. IGS is a distinguished professor and university health research chair, University of Ottawa. $\mathrm{CH}$ is supported by a mentored clinician scientist award from the Vancouver Coastal Health Research Institute. Competing interests: All authors have completed the ICMJE uniform disclosure form at www.icmje.org/coi_disclosure.pdf (available on request from the corresponding author) and declare: no support from any organisation for the submitted work; no financial relationships with any organisations that might have an interest in the submitted work in the previous three years; no other relationships or activities that could appear to have influenced the submitted work.

Ethical approval: The study protocol was approved by the research ethics boards at each centre without the need for written informed consent as patients continued to be investigated as per current practice. Participants were informed that they could be contacted by telephone for an update on their status, and verbal consent was obtained at the time of the telephone call. 


\section{What is already known on this topic}

Emergency patients presenting with sudden severe headache might have a subarachnoid haemorrhage, despite being neurologically intact at initial presentation

Traditionally, lumbar puncture has been done to exclude subarachnoid haemorrhage, even if results of initial computed tomography are negative

Conversely, as nearly all sudden headaches are benign and self limiting, universal investigation is inefficient and often involves unnecessary lumbar puncture

\section{What this study adds}

Modern thin sliced computed tomography is highly sensitive for subarachnoid haemorrhage when performed within six hours of headache onset and interpreted by an experienced radiologist

Computed tomography has unacceptably low sensitivity for subarachnoid haemorrhage when it is performed after six hours from the time of headache onset

Data sharing: No additional data available.

1 Edlow JA, Panagos PD, Godwin SA, Thomas TL, Decker WW. Clinical policy: critical issues in the evaluation and management of adult patients presenting to the emergency department with acute headache. Ann Emerg Med 2008;52:407-36.

2 Vermeulen M, van Gijn J. The diagnosis of subarachnoid haemorrhage. J Neurol Neurosurg Psychiatry 1990;53:365-72

3 Hop JW, Rinkel GJ, Algra A, van Gijn J. Case-fatality rates and functional outcome after subarachnoid hemorrhage: a systematic review. Stroke 1997;28:660-4.

4 Weir B. Headaches from aneurysms. Cephalalgia 1994;14:79-87.

5 Suarez JI, Tarr RW, Selman WR. Aneurysmal subarachnoid hemorrhage. N Engl J Med 2006;354:387-96.

6 Van Gijn J, Kerr RS, Rinkel GJ. Subarachnoid haemorrhage. Lancet 2007;369:306-18. Schievink WI. Intracranial aneurysms. N Engl J Med 1997;336:28-40.

8 Brisman JL, Song JK, Newell DW. Cerebral aneurysms. N Engl J Med 2006;355:928-39.

9 Edlow JA, Caplan LR. Avoiding pitfalls in the diagnosis of subarachnoid hemorrhage. $N$ Engl J Med 2000;342:29-36.

10 Perry JJ, Stiell IG, Wells GA, Spacek AM. Historical cohort study: "Use and yield of investigations for alert patients with possible subarachnoid hemorrhage." CJEM 2002;4:333-7.

11 Sidman R, Connolly E, Lemke T. Subarachnoid hemorrhage diagnosis: lumbar puncture is still needed when the computed tomography scan is normal. Acad Emerg Med 1996;3:827-31.

12 Sames T, Storrow A, Finkelstein J, Magoon M. Sensitivity of new-generation computed tomography in subarachnoid hemorrhage. Acad Emerg Med 1996;3:16-20.

13 Morgenstern LB, Luna-Gonzales $\mathrm{H}$, Huber JC Jr, Wong SS, Uthman MO, Gurian JH, et al. Worst headache and subarachnoid hemorrhage: prospective, modern computed tomography and spinal fluid analysis. Ann Emerg Med 1998;32:297-304.

14 Van der Wee N, Rinkel GJE, Hasan D, van Gijn J. Detection of subarachnoid haemorrhage on early CT: is lumbar puncture still needed after a negative scan? $J$ Neurol Neurosurg Psychiatry 1995;58:357-9.

15 Adams HP, Kassell NF, Torner JC, Sahs AL. CT and clinical correlations in recent aneurysmal subarachnoid hemorrhage: a preliminary report of the cooperative aneurysm study. Neurology 1983;33:981-8.

16 Tsementzis SA, Hitchcock ER, DeCothi A, Gill JS. Comparative studies of the diagnostic value of cerebrospinal fluid spectrophotometry and computed tomographic scanning in subarachnoid hemorrhage. Neurosurgery 1985;17:908-12.
17 Byyny RL, Mower WR, Shum N, Gabayan GZ, Fang S, Baraff LJ. Sensitivity of noncontrast cranial computed tomography for the emergency department diagnosis of subarachnoid hemorrhage. Ann Emerg Med 2008;51:697-703.

18 Boesiger BM, Shiber JR. Subarachnoid hemorrhage diagnosis by computed tomography and lumbar puncture: are fifth generation $\mathrm{CT}$ scanners better at identifying subarachnoid hemorrhage? J Emerg Med 2005;29:23-7.

19 Lourenco AP, Mayo-Smith WW, Tubbs RJ, Sidman R. Does 16-detector computed tomography improve detection of non-traumatic subarachnoid hemorrhage in the emergency department? J Emerg Med 2009;36:171-5.

20 Gee CA, Phanthavady T, McGuire T, Madsen T. New generation CT scanners demonstrate higher sensitivity for subarachnoid hemorrhage. Ann Emerg Med 2009;54:S12-3.

21 Saini S. Multi-detector row CT: principles and practice for abdominal applications. Radiology 2004;233:323-7.

22 Perry JJ, Stiell IG, Sivilotti ML, Bullard MJ, Lee JS, Eisenhauer M, et al. High risk clinical characteristics for subarachnoid haemorrhage in patients with acute headache: prospective cohort study. BMJ 2010;341:c5204.

23 Teasdale G, Jennett B. Assessment of coma and impaired consciousness. A practical scale. Lancet 1974;2:81-4.

24 Macdonald A, Mendelow AD. Xanthochromia revisited: a re-evaluation of lumbar puncture and CT scanning in the diagnosis of subarachnoid haemorrhage. J Neurol Neurosurg Psychiatry 1988;51:342-4.

25 Perry JJ, Sivilotti MLA, Stiell IG, Wells GA, Raymond J, Mortensen M, et al. Should spectrophotometry be used to identify xanthochromia in the cerebrospinal fluid of alert patients suspected of having subarachnoid hemorrhage? Stroke 2006;37:2472.

26 Perry JJ, Eagles D, Clement CM, Brehaut J, Kelly AM, Stiell IG. An international study of emergency physicians' practice for acute headache management and the need for a clinical decision rule. CJEM 2009;11:516-22.

27 Edlow JA, Wyer PC. Evidence-based emergency medicine/clinical question. How good is a negative cranial computed tomographic scan result in excluding subarachnoid hemorrhage? Ann Emerg Med 2000;36:507-16.

28 Cortnum S, Sorensen P, Jorgensen J. Determining the sensitivity of computed tomography scanning in early detection of subarachnoid hemorrhage. Neurosurgery .2010:66:900-2.

29 Vermeulen MJ, Schull MJ. Missed diagnosis of subarachnoid hemorrhage in the emergency department. Stroke 2007;38:1216-21.

30 Edlow JA, Bruner KS, Horowitz GL. Xanthochromia. Arch Pathol Lab Med 2002;126:413-5.

Accepted: 13 May 2011

Cite this as: BMJ 2011;343:d4277 


\section{Tables}

Table 1 Characteristics of $\mathbf{3 1 3 2}$ enrolled patients with acute headache in study of computed tomography for diagnosis of subarachnoid haemorrhage. Figures are numbers (percentage) of patients unless stated otherwise

\begin{tabular}{lc} 
Characteristics & No of patients $(\mathrm{n}=\mathbf{3 1 3 2})$ \\
Mean (SD) age (years) & $45.1(17.1)$ \\
\hline Women & $1889(60.3)$ \\
\hline Mean (SD) pain severity at peak $(0-10)(\mathrm{n}=2838)$ & $8.7(1.6)$ \\
\hline Worst headache of life $(\mathrm{n}=3065)$ & $2571(82.1)$ \\
\hline Onset during exertion $(\mathrm{n}=3103)$ & $381(12.2)$ \\
\hline Onset during sexual activity $(\mathrm{n}=3093)$ & $216(6.9)$ \\
\hline Transient loss of consciousness $(\mathrm{n}=3118)$ & $192(6.1)$ \\
\hline Awoken from sleep $(\mathrm{n}=3091)$ & $571(18.2)$ \\
\hline Neck painful/stiff by history $(\mathrm{n}=3094)$ & $1142(36.5)$ \\
\hline Vomiting $(\mathrm{n}=3096)$ & $922(29.4)$ \\
\hline Mean $(\mathrm{SD})$ heart rate (BPM) $(\mathrm{n}=3102)$ & $79.4(15.3)$ \\
\hline Mean (SD) blood pressure $(\mathrm{mm} \mathrm{Hg}):$ & $143.2(24.6)$ \\
\hline Systolic $(\mathrm{n}=3111)$ & $82.1(13.8)$ \\
\hline Diastolic $(\mathrm{n}=3108)$ & $180(5.7)$ \\
\hline Neck stiffness on examination $(\mathrm{n}=2902)$ & $755(24.1)$ \\
\hline Arrived by ambulance
\end{tabular}

BPM=beats per minute.

*No of patients with data recorded shown when characteristic not recorded for all patients. 
Table 2| Management and outcomes of $\mathbf{3 1 3 2}$ patients with acute headache in study of computed tomography for diagnosis of subarachnoid haemorrhage

\begin{tabular}{|c|c|}
\hline Outcome & No $(\%)$ of patients $(n=3132)$ \\
\hline \multicolumn{2}{|l|}{ Diagnostic procedures: } \\
\hline Computed tomography $\leq 6$ hours after headache onset & $953(30.4)$ \\
\hline Lumbar puncture & $1546(49.4)$ \\
\hline Computed tomography angiogram & $229(7.3)$ \\
\hline Traditional angiogram & $75(2.4)$ \\
\hline Magnetic resonance imaging angiogram & $13(0.4)$ \\
\hline \multicolumn{2}{|l|}{ Final diagnosis: } \\
\hline Benign headache & $1750(55.9)$ \\
\hline Migraine & $629(20.1)$ \\
\hline Subarachnoid haemorrhage & $240(7.7)$ \\
\hline Viral illness & $90(2.9)$ \\
\hline Acute ischaemic stroke/transient ischaemic attack & $65(2.1)$ \\
\hline Postcoital headache & $55(1.8)$ \\
\hline Sinusitis & $36(1.1)$ \\
\hline Syncope & $33(1.1)$ \\
\hline Neck strain & $13(0.4)$ \\
\hline Brain tumour with mass effect & $9(0.3)$ \\
\hline Subdural haematoma & $9(0.3)$ \\
\hline Intracerebral haemorrhage & $8(0.3)$ \\
\hline Hypoglycaemia & $4(0.1)$ \\
\hline Weakness-not determined & $4(0.1)$ \\
\hline Bacterial meningitis & $1(0.03)$ \\
\hline Other benign cause* & $186(5.9)$ \\
\hline Admitted to hospital & $345(11.0)$ \\
\hline
\end{tabular}

*No patients were admitted to hospital or had diagnosis with any risk of important morbidity or mortality. 
Table 3| Sensitivity of computed tomography for subarachnoid haemorrhage in patients with acute headache stratified by timing of scan

\begin{tabular}{|c|c|c|c|c|c|c|c|}
\hline \multirow{2}{*}{$\begin{array}{l}\text { Time from } \\
\text { headache onset to } \\
\text { scan }\end{array}$} & \multirow{2}{*}{$\begin{array}{c}\text { No of } \\
\text { patients }\end{array}$} & \multirow[b]{2}{*}{$\%$ Sensitivity (95\% Cl) } & \multirow{2}{*}{$\begin{array}{l}\% \text { Specificity }(95 \% \\
\text { CI) }\end{array}$} & \multicolumn{2}{|c|}{ Likelihood ratio $(95 \% \mathrm{Cl})$} & \multicolumn{2}{|c|}{ Predictive value $(95 \% \mathrm{Cl})$} \\
\hline & & & & Positive & Negative & Positive & Negative \\
\hline All patients & 3132 & 92.9 (89.0 to 95.5$)$ & 100 (99.9 to 100$)$ & Infinity & 0.07 (0.05 to 0.11$)$ & $100(98.3$ to 100$)$ & 99.4 (99.1 to 99.6$)$ \\
\hline$\leq 6$ hours & 953 & $100(97.0$ to 100.0$)$ & $100(99.5$ to 100$)$ & Infinity & 0.00 (0.00 to 0.02$)$ & 100 (96.9 to 100$)$ & $100(99.5$ to 100$)$ \\
\hline$>6$ hours & 2179 & 85.7 (78.3 to 90.9$)$ & 100 (99.8 to 100$)$ & Infinity & $0.14(0.14$ to 0.17$)$ & $100(96.3$ to 100$)$ & 99.2 (98.7 to 99.5$)$ \\
\hline
\end{tabular}




\section{Figure}

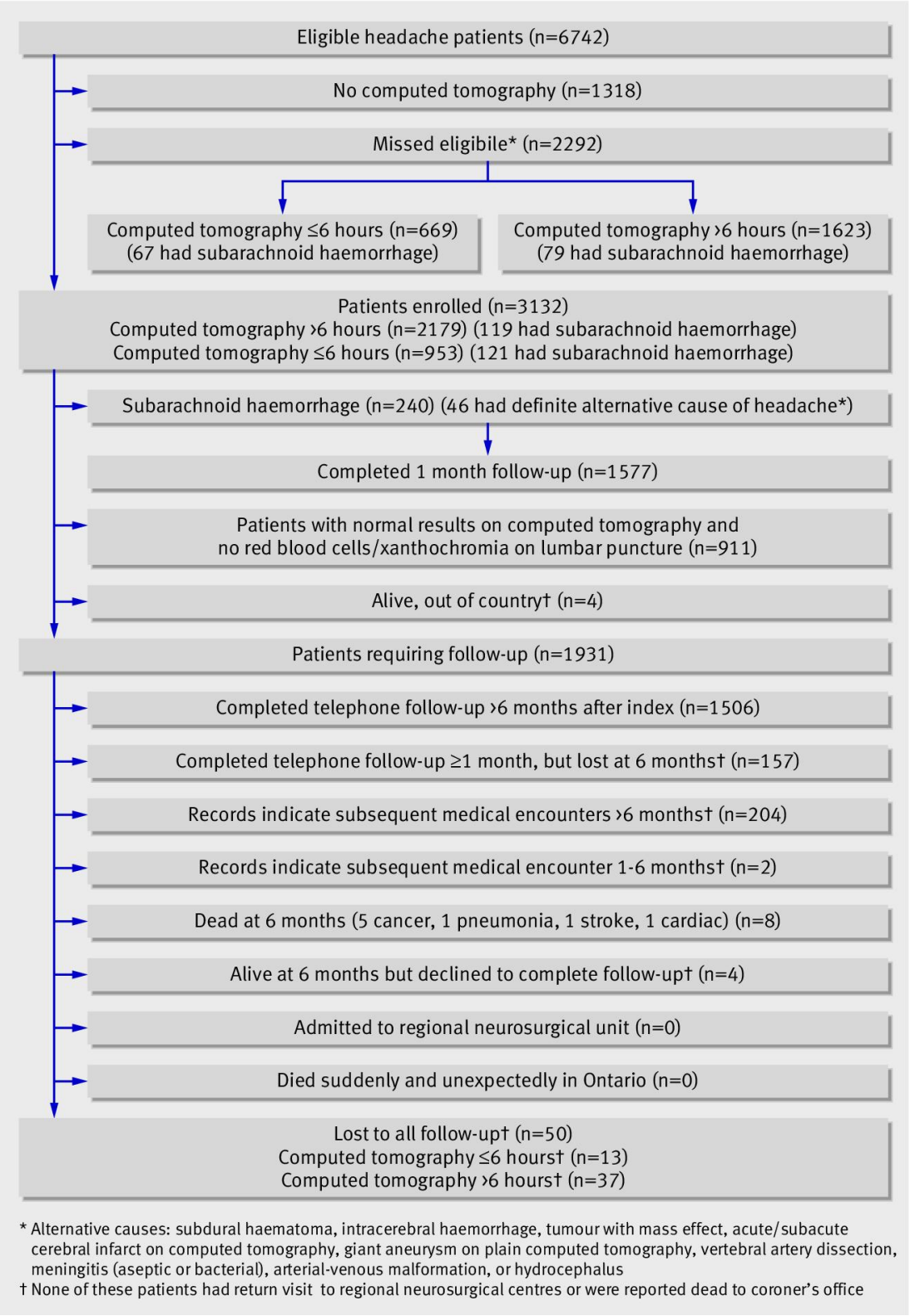

Flow and follow-up of patients in study of computed tomography for diagnosis of subarachnoid haemorrhage 